\title{
Colored aftereffects contingent on patterns generated by Lie transformation groups
}

\author{
VICTOR F. EMERSON, G. KEITH HUMPHREY, and PETER C. DODWELL \\ Queen's University, Kingston, Ontario, Canada
}

\begin{abstract}
Two experiments are described which make a preliminary test of the power of the Lie transformation group model of neuropsychology (LTG/NP) in predicting pattern-specificity of visual aftereffects of color. In Experiment 1, each of three groups of eight subjects reported contingent aftereffects (CAEs) of color following induction with one of the three pairs of static representations of first-order Lie orbits, these patterns having been presented alternately (one in red light and one in green) every $9.5 \mathrm{sec}$ during a 10-min adaptation period. This result demonstrates that CAEs can be subserved by each of the three pairs of elementary patterns generated by the LTG/NP. In Experiment 2, induction occurred to all three pairs of elementary Lie patterns in sequence. Eleven of the 12 subjects reported color aftereffects, five subjects on patterns from all three pairs, indicating that several pattern-specific aftereffects of color can coexist in an individual observer. Together, these results suggest that the LTG/NP is of value in describing the mechanism of CAEs, which are taken here to be subserved not by visual "molecules," such as edges or dipoles, but rather by global vectorfield properties of patterns.
\end{abstract}

One of the most prominent recent discoveries in visual science is the orientation-contingent color aftereffect, commonly known as the McCollough effect. An observer who views, alternately, a horizontal grating in green light and a vertical grating in red light, for a total induction period of several minutes, will later report seeing on an achromatic test pattern a pinkish hue on horizontal stripes and a greenish tinge on vertical stripes (McCollough, 1965). The effect demonstrates that the human visual system can store chromatic information for prolonged periods if the chromatic input is paired with distinctive patterns, but there has been widespread disagreement about the sorts of pattern with which McCollough effects can be generated, and about the mechanism underlying such contingent aftereffects (CAEs).

McCollough's experiment first demonstrated that orthogonal orientation of lines in the inspection patterns was a sufficient condition for producing the effect. She also reported that it was the retinal orientation of the lines that was important, the apparent colors reversing on the test pattern if the head was tilted $90^{\circ}$ to one side. It has since been shown that the strength of the McCollough effect is reduced if the gratings are orthogonal during induction but have a smaller angular separation in testing (Teft \& Clark, 1968) or if nonorthogonal gratings are used in in-

This work was supported by Grant A0044 of the Natural Sciences and Engineering Research Council of Canada to P. C. Dodwell and D. W. Muir. We share authorship equally. After completion of these studies, we learned that K. D. White had used similar patterns in earlier, but unpublished studies. V. Emerson is now at 562 Fraser Avenue, Ottawa, Ont. Canada, K7A 2R4. G. K. Humphrey is now at the Department of Psychology, University of Lethbridge, Lethbridge, Alta., Canada, T1K 3M4. Reprint requests should be sent to P. C. Dodwell at: Department of Psychology, Queen's University, Kingston, Ont., Canada K76 3N6. duction (Fidell, 1970). McCollough originally proposed that the mechanism of this CAE involved color adaptation of "edge detectors" like those known to exist in the visual cortex of cats (Hubel \& Wiesel, 1962) and, later, monkeys (Hubel \& Wiesel, 1968). Harris and Gibson countered (1968) that the orientation-selective CAE could be generated by more basic coding units which they called dipoles. Although such hypothetical units can account for a remarkable range of CAE phenomena, they cannot account for CAEs based on nonlinear stimuli such as angles and curves (Riggs, 1973; White \& Riggs, 1974). After reviewing the evidence on the sorts of patterns that might generate CAEs, Stromeyer (1978), in a major review of the field, concluded that:

It thus appears quite difficult to obtain color aftereffects that are selective to even relatively simple patterns like curves and angles. This strongly suggests that the diversity of the mechanisms underlying the form-color aftereffects may be quite limited, and the colored aftereffects may thus reflect a process of adaptation or conditioning of rather elementary visual units (p. 109).

Stromeyer's review covers the controversy in the literature concerning the ability of more complex patterns involving angles and curves to generate or subserve CAEs. The evidence is quite strong that they can do so (e.g., Leppmann, 1973; White \& Riggs, 1974); what is uncertain is whether such curve- and angle-contingent effects can be explained solely on the basis of local adaptation of oriented line detectors. In many cases, they probably can; however, evidence is provided below that CAEs based on stationary patterns are generated not by line and edge detectors, or by more elementary units such as dipoles, but rather by a more global mechanism, probably operating at the level at which pattern information 
itself is processed. The distinction between pattern element coding, such as that discovered by neurophysiological investigation of single units, and the elaboration of patterns, which in some sense entails the integration of information from such local elements, is discussed at length by, for example, Dodwell (1970b, 1978) and Marr (1982). It has been widely held that CAEs are generated at the level of pattern element coding. It is shown below that this cannot, in general, be true.

The idea that a McCollough effect might be generated at a molecular level by such units as edge detectors or dipoles has been attacked in the past from two points of view. The first points out that the time course of adaptation and recovery for these effects is quite different from the time course of neurophysiological adaptation and recovery; whereas the latter is typically measured on the scale of milliseconds or at most minutes, the McColloughtype contingent aftereffect may last for weeks (Skowbo, Gentry, Timney, \& Morant, 1974) or even months (Jones $\&$ Holding, 1975). There is no evidence that simple neurophysiological units adapt or recover on this time scale. The second criticism originates in the proposal by several writers that the mechanism involved is a form of coding based on space-invariant, linear, spatial-frequency and orientation-selective filters (Breitmeyer \& Cooper, 1972; Green, Corwin, \& Zemon, 1976; Lovegrove \& Over, 1972; May \& Matteson, 1976; Stromeyer, 1972b; Wyatt, 1974). However, this filter model is incomplete in that such filters might themselves be constructed out of dipoles, and other interpretations of spatial-frequencyspecific effects, invoking a simpler receptive field model, have not been ruled out (Smith, 1977; Tyler, 1977).

The extreme form of the filter hypothesis is that the visual system performs a crude form of Fourier analysis on the visual input. Whether or not the Fourier analyzing hypothesis is correct, it is clear that analyzers sensitive to spatial frequency respond not just to the orientation of a single edge in a particular location, but also to the properties of the "neighborhood" of that edge. A previously unexplored possibility is that such neighborhood analysis is based not on spatial frequency information as such, but rather on the extended vectorfield properties of the visual manifold, of which edge and line detectors are themselves elements.

The concept of vectorfield analysis in vision is certainly not new (e.g., Mach, 1886/1959), but only recently was an attempt made to apply the mathematics of vectorfield operation to vision on a broad scale (Hoffman, 1966, 1977). This ambitious theoretical model, now known as the Lie transformation group model of neuropsychology (LTG/NP), is based on Lie's theory of continuous transformation groups (Lie \& Engels, 1888). LTG/NP postulates that the human visual system has not evolved in isolation from the environment in which it operates, and so continuous transformation groups that are related to the invariances (constancies) of visual perception play a fun- damental role in perception and are structurally embedded in the visual cortex in an anatomical representation of the visual manifold. The theoretical structures involved, the so-called Lie orbits, are simple sets of "integral curves" on the visual manifold which can manifest themselves as sets of visual contours of a particular type. According to LTG/NP, they are basic to all pattern processing.

There are three pairs of basic patterns in what has been called the "primitive model" of LTG/NP (Dodwell, 1983). These, shown in Figure 1a, all show very high degrees of symmetry, albeit different forms of symmetry in each of the three pairs. If superimposed on each other, the two patterns in each pair would constitute families of orthogonal orbits; that is, the contours in one pattern would cut those of the second member of the pair at right angles at every point. This is a fundamental property of such basic orbits in LTG/NP. This orthogonality is clearly both a local and a global property of the pattern pairs.

Two experiments were conducted to assess the usefulness of the three pairs of patterns shown in Figure la as induction patterns in a standard McCollough-type design. The aim of the first experiment was to ascertain whether whole-field, pattern-specific, pattern-contingent aftereffects could be obtained with the Lie patterns. If they could, then one could argue that the "elementary visual units" referred to by Stromeyer (1978) cannot be defined merely in terms of edge orientation or spatial frequency, and consequently local orientation-specific coding must be an insufficient basis for understanding these sorts of effects. The patterns used in the studies reported here are simple, from the point of view of Hoffman's model, because they are generated by first-order Lie group operators and have a simple mathematical description, but they are not of equivalent complexity in any other theory of pattern perception yet proposed.

It is clear that visual patterns vary in their ability to subserve CAEs, but the nature of this variability is unknown; thus, it is not possible at present to refer to a model or metric that permits one to predict which patterns may, and which may not, subserve CAEs. The present experiments were designed to test the hypothesis that LTG/NP provides such a model. It was assumed that the effectiveness of a pattern in subserving a CAE was related to the pattern's simplicity, that is, the extent to which the pattern reflected some fundamental organizational principle of the nervous system. From this one may predict that the first-order Lie orbits, being the fundamental structures of LTG/NP, should all be effective in generating CAEs; ideally, their comparable complexity in LTG/NP would translate into a corresponding perceptual complexity. The first experiment tested this prediction in a betweensubjects design, using orthogonal pairs of first-order Lie orbits (Figure 1a) as adapting patterns in the usual McCollough paradigm. The first experiment aimed simply to determine whether each pair of orthogonal Lie patterns could subserve CAEs in individual subjects. 


\section{EXPERIMENT 1 CAEs WITH LIE PATTERNS}

\section{Method}

Subjects. Twenty-four female undergraduates, naive with respect to the experiment, served as subjects. All had normal or correctedto-normal vision.

Apparatus and Materials. Each of the patterns shown in Figure la (the inducing patterns) was mounted in a slide holder with either a Kodak Wratten 92 (red) or 74 (green) filter, and these were projected from a Kodak Carousel projector. A Gerbrands twochannel projection tachistoscope (Series 300 ) control unit drove two shutters regulating the output from the projectors so that each stimulus was on for $9.5 \mathrm{sec}$ with a 5 -msec delay between stimuli. The displays, which were black except for the colored bars, were projected onto a plain white screen, each pattern subtending $11.5^{\circ}$ of visual angle at the viewing distance of $120 \mathrm{~cm}$. The spatial frequency of the gratings was about 1 cycle/degree of visual angle. In addition, there was a series of high-contrast matte photographs of the six test patterns shown in Figure 1b. Each test pattern was a composite of the two inducing patterns comprising an orthogonal pair, and the two test patterns in a pair exhausted the respective inducing patterns.

Procedure. Subjects were tested individually in a three-stage procedure; pretest, induction, and posttest. The 24 subjects were divided into three groups of 8; each group was induced with one of the three pattern pairs.

First, each subject was shown the six achromatic test patterns of Figure $1 \mathrm{~b}$ and asked to name colors seen on them, if any. The subjects reporting colors in this pretest session were replaced. Thus,
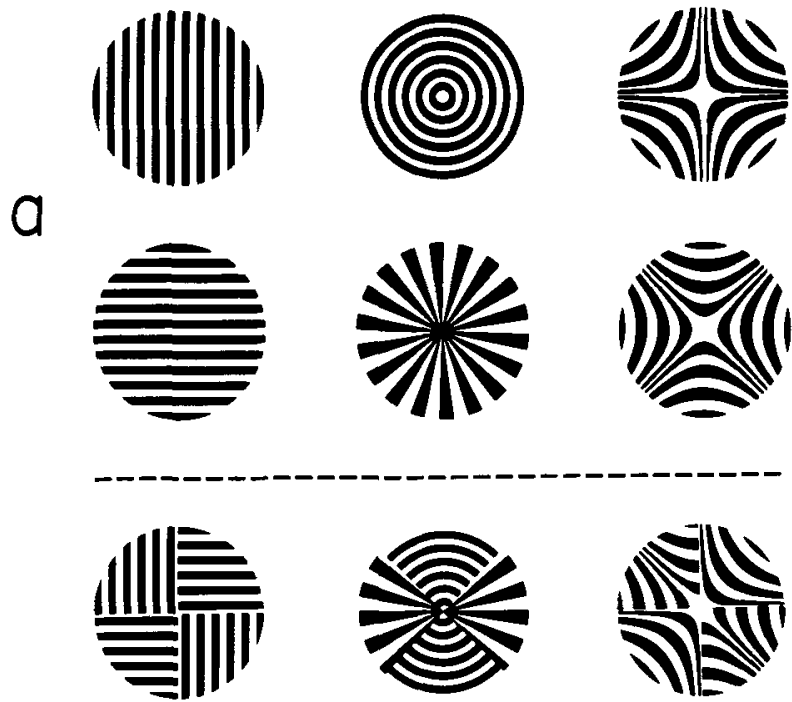

○
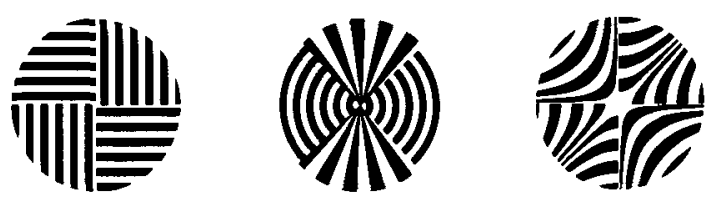

Figure 1. (a) The three pairs of elementary Lie orbits used as inducing patterns. The two patterns within each pair are orthogonal. Each subject adapted to one pair in Experiment 1 and to all three pairs in Experiment 2. (b) The three pairs of test patterns. Each pattern comprises alternating quadrants from the two inducing patterns in one pair. All subjects in Experiments 1 and 2 were tested (and pretested) on all six patterns. none of the subjects whose data are reported in either Experiment 1 or 2 reported seeing any colors in the pretest session. Immediately following this pretest, a 10-min induction session was given in which the red and the green patterns were presented alternately in a darkened room. The inducing stimuli were always the relevant pair of Lie orbits, four subjects in each group having one color-pattern contingency, such as red-vertical, green-horizontal, and the other four having the alternative contingency. The subject was instructed to keep her head in a fixed position during induction and also to maintain her fixation on the center of the inducing pattern. ${ }^{1}$

Following induction, there was a 5 -min rest period in normal room light, followed by the posttest, in which the subject was given a pen and a booklet containing photocopies of the six test patterns (Figure 1b), one pattern per page. She was shown a succession of high-contrast matte photographs of the six test patterns representing all three pairs of inducing stimuli, and told to fixate the center of each pattern in turn. These were presented so as to subtend the same visual angle at the subject's eye as had the inducing pattern. The subject was asked to report any colors seen on the test patterns by outlining, as accurately as possible, the appropriate "colored" area on the corresponding pattern in the booklet, writing down also the name of the color seen. No color names were suggested to her, and it was made clear that she might not see any colors. The order of presentation of the test patterns was varied across subjects.

Scoring. Because each test pattern was a composite of quadrants from the two inducing patterns in a pair, the subject gave two responses per test pattern, one per contributing induction pattern. For each, the drawings were scored either as "complete" or "partial" aftereffects. A response was scored as "complete" only if the subject outlined completely in a single test pattern, both quadrants that had come from a single induction pattern. For example, if the subject outlined both "vertical" quadrants of the pattern in the upper left corner of Figure $1 \mathrm{~b}$, that response would be scored as "complete." If only one quadrant were outlined, or parts of one or both quadrants, the response would be categorized as "partial." This dichotomous classification scheme attempted to distinguish between "global" aftereffects and those which might be attributed to more local induction. In addition, each report was assigned a positive sign for a correct (complementary) hue or a negative sign for an incorrect hue.

\section{Results}

An appropriate (complementary) CAE to at least one of the inducing patterns was reported by each subject; only one (partial) CAE of an inappropriate color was reported. The pattern of responding, shown in Table 1, was that complete reports were given to the patterns on which the subject had been induced, partial CAEs being reported for the other patterns, on which the subject was tested but on which she had not been induced. This pattern of reporting complete CAEs is shown in greater detail in Table 2.

With reference to Table 1, although the categories of complete and partial are mutually exclusive, the entries are not strictly independent, for an individual subject could give a maximum of two CAE reports to each of the two induced patterns (in any combination of "complete" and "partial" reports) plus two to each of the four noninduced patterns (total $=12$ ). Nevertheless, there is a pronounced tendency $\left[\chi^{2}(1)=95.54, p<.001\right]$ for complete CAE reports to be associated with the patterns used in induction ( 59 of 72 reports $=81.9 \%$ ) and the partial reports to be linked to patterns not so used (113 of 129 reports $=87.6 \%$ ). This tendency was equally strong across in- 
Table 1

Assignment of 201 CAE Reports (Experiment 1) as a Function of the Spatial Extent of the Report

\begin{tabular}{lcc}
\hline & \multicolumn{2}{c}{ CAE Report } \\
\cline { 2 - 3 } & Complete & Partial \\
\hline Induced Pattern & 59 & 16 \\
Noninduced Pattern & 13 & 113 \\
\hline
\end{tabular}

duction groups. The same dependence is evident if number of subjects reporting CAEs, rather than number of CAE reports, is used as the dependent measure $\left[\chi^{2}(1)=\right.$ 51.043, $\mathrm{p}<.001]$.

Table 2 shows the distribution of the 72 reports of complete CAEs. Fifty-nine of the 72 reports (81.9\%) occurred on the patterns on which the subject had been induced (principal diagonal); this number is entered in the upper left-hand corner of Table 1.

Thus, global CAEs were associated with patterns on which the subject had been induced, local CAEs being more commonly reported but with evidence of less pattern specificity. Rather, the local (partial) CAE reports tended to be assigned to those portions of the test patterns where the local orientation cue for a particular retinal locus matched what had been present in one of the inducing patterns. For example, analysis of these partial CAE reports following induction to the grating pair indicated that when local aftereffect colors "transferred" to the nongrating test patterns, they were assigned to contours whose orientations were within about $20^{\circ}$ of horizontal or vertical. ${ }^{2}$ Not too much emphasis should be placed on this result, since the appropriate psychophysical procedures for estimating tuning widths for orientation selectivity were not used, but the pattern of results suggests that global CAEs are specific to the inducing patterns and that local CAEs, which can occur quite frequently, tend to appear at those retinal loci where the test and inducing patterns are nearly congruent. Because of the relatively indiscriminate assignment of partial CAE reports to inducing patterns and the strong association of complete $\mathrm{CAE}$ reports with inducing patterns, partial CAE reports are not considered further.

A problem with using the number of CAE reports as the only dependent measure is that their number varies across subjects as well as across conditions. The extent of this variation is seen in Figure 2a, which shows, separately for each induction group, the number of patterns on which subjects reported complete CAEs. These distributions are shown, summed across induction groups, in Figure $2 b$. Although each subject was adapted to only two patterns, some reported complete CAEs on more than

Table 2

Distribution of 72 Reports of Complete CAEs (Experiment 1)

\begin{tabular}{lccc} 
& \multicolumn{3}{c}{ Induced On } \\
\cline { 2 - 4 } Tested With: & Gratings & Star/Bullseye & Hyperbolae \\
\hline Gratings & 31 & 5 & 1 \\
Star/Bullseye & 2 & 15 & 1 \\
Hyperbolae & 0 & 4 & 13 \\
\hline
\end{tabular}

two patterns. A total of 13 such "false alarms" was reported by 6 of the 24 subjects; the distribution of these is shown in Table 2 (all entries off the principal diagonal). In all cases but one, these "misplaced" CAE reports were accompanied by reports of complete CAEs on the inducing patterns. (The misplaced reports have been included in Figure 2 so that meaningful comparisons may be made with Figure 3a (below, Experiment 2), where it is not possible to separate "hits" from "false alarms.")

Although the results from the two groups induced with nongrating stimuli might seem weak compared to those of the group adapted to the gratings, it is worth noting that most previous investigators of McCollough-type effects have used yes/no reports or measures of the saturation of the CAE hue without attempting to determine the distribution of the CAE reports across the spatial extent of the pattern. In this sense, the results shown in Figure 2 are more conservative than those usually reported, presenting only the "complete" reports and eliminating the more local CAEs that might otherwise be counted as "yes" responses. Even when this conservative scoring strategy is used, at least $62.5 \%$ (5 out of 8 ) of the subjects in each group reported a complete CAE, a percentage comparable to that reported by other investigators. Although the present results show that gratings are the best of the inducing (or test) stimuli of those we used, the evidence is clear that all three pairs of orthogonal patterns can subserve McCollough-type effects.

\section{EXPERIMENT 2 MULTIPLE INDUCTION}

It is generally accepted that McCollough-type effects are retinally specific, in that they occur strongly only in the area of the visual field to which the inducing stimulation was applied (Stromeyer, 1972a). Central fixation was used during induction and testing in Experiment 1, and so the results cannot be taken as strong evidence that the CAEs reported here are pattern-specific in a global sense. Because each pair of Lie patterns contains local (as well as global) orthogonality, it might be argued that the reported CAEs represented chromatic induction only to the local orientation of a contour (for example, the tangent to a curved contour at a particular locus); in this case, the apparent global nature of the colored effects would be an artifact of the care with which central fixation was maintained. There has been considerable debate in the literature over the question of whether CAEs based on curvature and angle, for example, can be explained in terms of the local tangent orientations of the patterns used (Crassinin \& Over, 1975; Sigel \& Nachmias, 1975). To demonstrate whole-pattern specificity of the CAEs, it would be both necessary and sufficient to show that the induction was independent of local contour orientations within each pattern, but contingent on the specific pattern in which those local orientations occurred. The prediction that whole-field CAEs are contingent on patterns rather than merely on local contour orientation fol- 

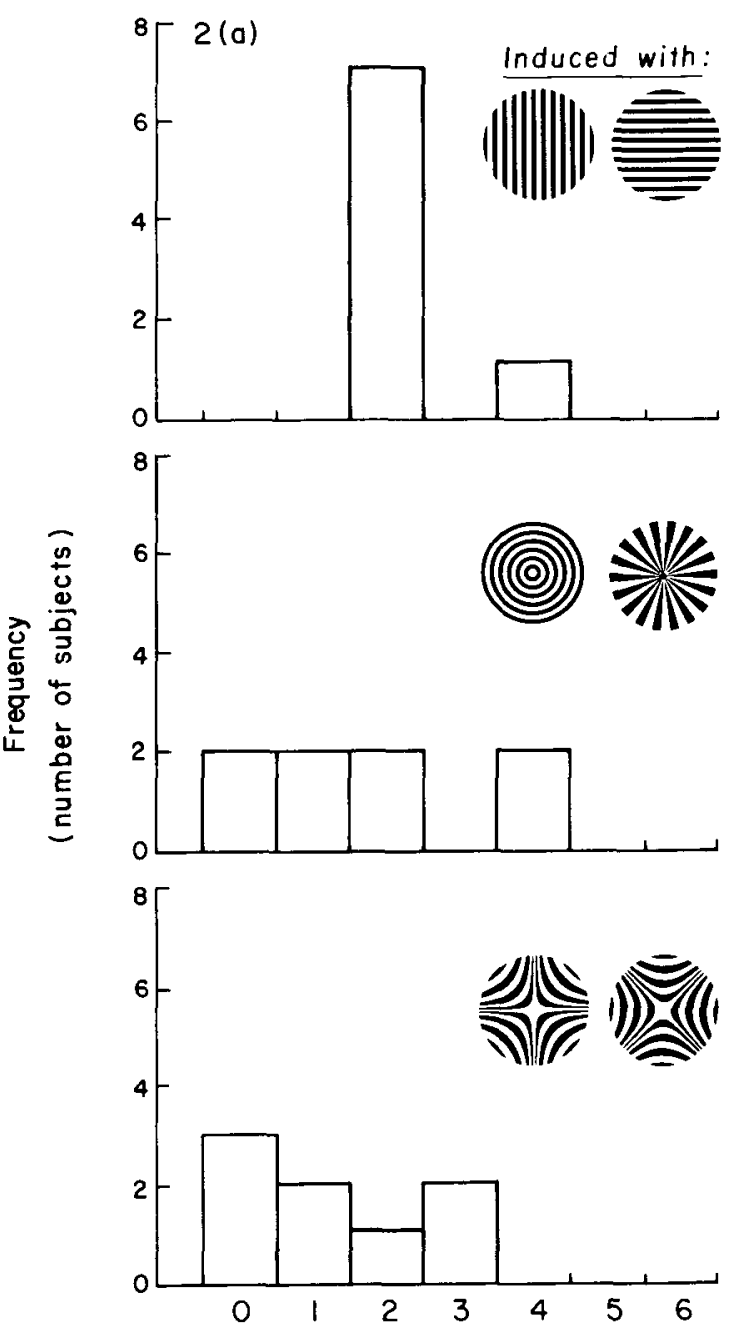

Number of Inducing Patterns on Which Complete CAEs Were Reported

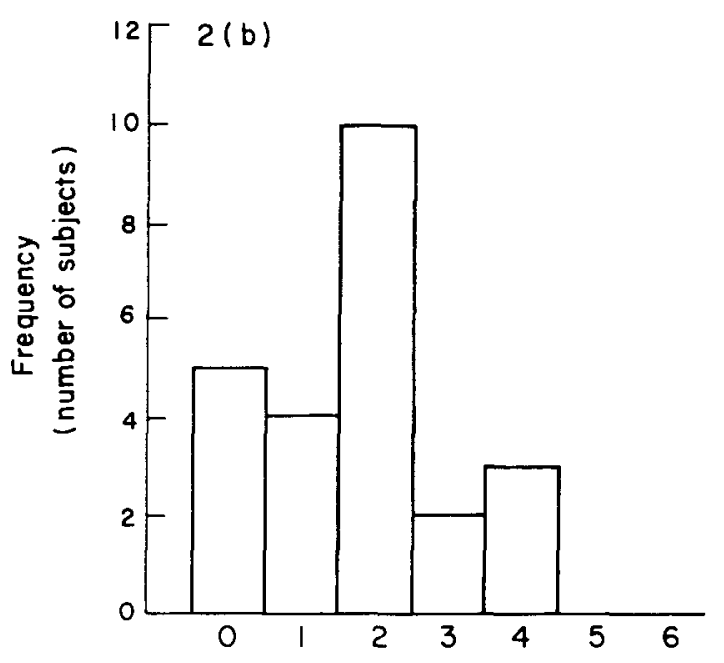

Number of Inducing Potterns on Which Complete CAEs Were Reported lows naturally from the interpretation of the CAEs as being generated by vectorfield properties.

According to Hoffman's LTG/NP model, the six patterns of Figure 1a are not only mutually orthogonal in pairs, as discussed earlier, but also constitute, in a somewhat broader sense, three different and independent "channels" for the processing of pattern information in the visual system. The independence of these three channels is postulated on the ground that the three pairs of transformations on which the Lie orbits are based are separate from each other, are generated by different local Lie operators, and arise because of three different sorts of ecological constraint on the active perceiving organism (Dodwell, 1983). If the three orbit pairs correspond to different and independent perceptual channels, then not only would each pair be able to sustain a CAE (as was demonstrated in Experiment 1), but there should also be a high degree of independence between the CAEs generated by each pair. It was therefore predicted that within an individual subject it should be possible to demonstrate simultaneously three different CAEs, each one generated by one of the three orbit pairs of Figure 1a. Their (relative) independence should be demonstrable in terms of lack of interference among CAEs generated by the three pairs. To demonstrate that the different CAEs should coexist in the same observer, each individual subject was induced with all three pairs of patterns (Figure 1a) in one extended three-part induction session.

\section{Method}

Subjects. Twelve female subjects, naive with respect to the experiment and with normal or corrected-to-normal vision, were paid to participate in this experiment.

Apparatus and Materials. These were the same as in Experiment 1.

Procedure. Following an initial pretest (described in Experiment 1), each subject was given three 10-min induction sessions of precisely the same sort as in Experiment 1, each one followed by a 5 -min rest period. During each 10 -min segment, the subject was induced with one of the three pairs of patterns (Figure la), the patterns being shown in red and green illumination, as before. After a 5-min rest, she was induced with another pattern pair, also in red and green, and so on for three inductions. Three groups of four subjects each were run, with the order of pattern pairs used for induction varying across groups and color-pattern contingency counterbalanced within each group. Following the final 5-min rest period, the subjects were given a posttest, as in Experiment 1.

\section{Results}

If induction could occur successfully to more than one pair of patterns, the number of patterns for which CAE reports were given should be greater than 2 . Because the results of Experiment 1 showed clearly that partial CAE reports are not as useful as complete reports in dis-

Figure 2. (a) Frequency distributions showing the number of patterns on which complete CAEs were reported, plotted separately for each inducing group in Experiment 1. (b) The summed frequency distributions of Figure $2 a$, showing the relative probability that a subject induced on one pair of orthogonal Lie patterns will report complete CAEs on 0-6 patterns. 

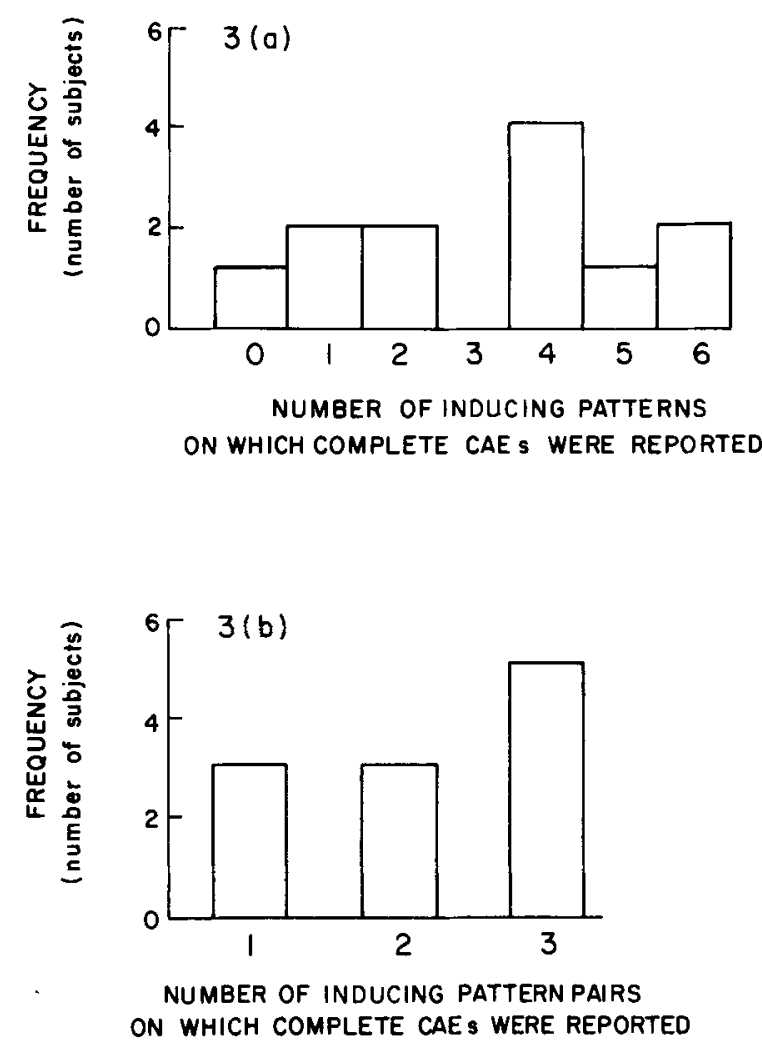

Figure 3. (a) Frequency distribution showing the number of patterns on which complete CAEs were reported by the 12 subjects in Experiment 2. (b) For the 11 subjects in Experiment 2 who reported complete CAEs on at least one pattern, the number of orthogonal pairs (cf. Figure 1a) which these patterns represented.

criminating between induced and noninduced patterns, only complete CAE reports are considered here.

Figure 3a shows that the number of inducing patterns for which complete CAEs were reported by each subject ranged from 0 (one subject) to 6 (two subjects), the mode and median being 4 and the mean 3.25 . The mean of this distribution is significantly greater than the mean (1.75) of the cumulative distribution of CAE reports obtained in Experiment 1 (Figure 2b) $[\mathrm{t}(34)=2.753, \mathrm{p}<.005$, one-tailed), indicating that the multiple induction procedure employed here increased the number of complete CAE reports.

Figure $3 b$ shows the distribution of these $\mathrm{CAE}$ reports across inducing pattern pairs, and shows that 5 of the 12 subjects reported at least one complete CAE from all three pairs and 8 of the 12 subjects reported CAEs from at least two. As in Experiment 1, every complete CAE report was of the appropriate hue. Thus, although not all subjects showed evidence of multiple induction in this condition, it is clear from the positive results that several color aftereffects contingent on pattern can coexist in an individual observer and that induction with more than one pair of patterns increases the frequency of CAE reports.
The complete CAEs were both pattern-specific and of the correct type; this is emphasized by subjects' reports that, for example, a horizontal contour in one part of a particular pattern (say, near the left edge) would be reported as having an appropriate pinkish tint if it was part of a horizontal grating or the $0^{\circ} / 90^{\circ}$ hyperbolae, but an appropriate green tint if it formed part of the radial pattern. Clearly, because the local vector orientation is the same for all three patterns in such cases, the obtained CAE is pattern-specific in the sense that its hue is determined by the context of the whole pattern rather than the local contour orientations alone.

\section{GENERAL DISCUSSION}

The present findings demonstrate that stimuli other than gratings, checkerboards, and other simple patterns can be used to generate CAEs. (By "simple" is meant any pattern in which the CAE can be attributed solely to the coloring of a local straight edge or tangent to a curve.) To quote again from Stromeyer's (1978) review:

The form-color aftereffects can readily be made selective to rather simple form properties, like the orientation, width, and velocity of lines. At present it is questionable whether aftereffects can be made selective to more complex patterns. For example, it appears most difficult to obtain color aftereffects selective to the convexity, or curvature, of lines . . . [T]hese properties suggest that the form-color aftereffects depend on the modifications of relatively simple visual units that are driven by one eye (p. 135).

The present experiments have shown that the mechanism of the CAE must involve something more than lower order units that are sensitive only to luminance differences across an edge or contour. Rather, the mechanism must use a form of global information from higher order processes capable of recognizing a particular local contour in the context of a larger pattern to which it belongs (see also Jordan \& Uhlarik, 1983, on the role of context in CAEs).

Other investigators have attempted, without success, to generate CAEs using "complex" patterns (e.g., Fidell, 1968); why, then, have the present experiments been more successful? It seems relevant that the patterns used in the present experiments were chosen not simply because they were symmetrical, smoothly curved, high-contrast, and "redundant," but also because they play a very special role within a particular theoretical framework. Because these patterns can successfully subserve CAEs, the results provide support for the model from which the patterns are derived. Hoffman's LTG/NP model demonstrates the mathematical simplicity of the description of these "primitive" orbit pairs; although the present findings lend specific support to this model, they also address a wider issue. It is evident that not all McCollough-type CAEs can be explained completely in terms of the chromatic adaptation of local pattern elements. Any model based on 
such a principle would have difficulty explaining how a contour at a specific position and orientation can on one occasion manifest one color contingency, but on another occasion the opposite. In the present experiments, the whole pattern context determines the chromatic bias, and in the present interpretation, pattern context is supplied by the underlying vectorfield properties. Certain simple vectorfield properties are manifested (at least partially) in the patterns used here. As others have remarked (e.g., White \& Riggs, 1974), not just any pattern can be used to induce McCollough-type effects; different faces or makes of automobile, for example, will not do. Clearly, then, it is important to consider the question of which vectorfield properties are the relevant ones.

The present results are neither unique nor original in showing that the LTG/NP orbits can be used to generate McCollough effects. Curiously, in a footnote in her original paper, McCollough (1965) stated that "MacKay's figures 1 to 3 are excellent patterns for observing these color aftereffects." Two of those patterns are the radial lines and bulls-eye of Figure 1a; this "sleeper" has been in the literature for nearly 20 years. Nor is the present paper the first to report multiple CAEs; MacKay and MacKay (1977) have demonstrated that several aftereffects contingent on line orientation can be generated successively in individual observers. Rather, the contribution made by the present report is one of theory, since a somewhat novel approach (LTG/NP) has been used to account for previous findings and generate hypotheses which led, in the present studies, to findings consistent with the predictions generated by the Lie theory. Thus, the use of this theoretical framework enables one to predict with some confidence at least some patterns that can generate CAEs and, by inference, the level of processing at which an explanation for CAEs should be sought.

The present findings support the notion that McCollough-type CAEs in general are mediated by vectorfields in the visual substrate of pattern processing. There are other, more direct methods for investigating vectorfield processing (see, e.g., Caelli \& Dodwell, 1982; Dodwell \& Caelli, 1985) that should be useful in elucidating the vectorfield basis for these effects. At the very least, it should be possible to determine the limits of the class of patterns capable of sustaining such CAEs.

Caelli and Dodwell (1985) show that certain "simple" vectorfields, namely those that embody a one-parameter transformation rule, have special properties as visual figures. In fact, one subset of such vectorfields comprises the primitive Lie orbits represented in the patterns of Figure 1a. A reasonable conjecture would be that any vectorfields of this sort, or their simple linear combinations, are capable of sustaining McCollough-type CAEs.

If this conjecture is correct, then one should be able to demonstrate a whole class of CAEs generated by smoothly varying patterns like those used here, even though they do not conform strictly to the Lie orbits of the LTG/NP primitive mode. It will be of some interest to know, through a more quantitative study, whether or not the primitive orbits hold a special place compared to other smooth and symmetrical patterns.

It has been reported that color CAEs demonstrate some of the properties of "anomalous transfer" (Dodwell, 1970a). That is to say, there are certain test figures that give stronger CAEs than the original inspection figures. The point is raised, and discussed, by Crassini, Broerse, and O'Shea (1979), particularly with regard to the finding of Sharpe and Tees (1978) that "complete" patterns (square-wave grids) are more effective than "fragmented" patterns, in which the main contour lines are only partially represented. A similar finding has been reported for CAEs in curved and angled figures, in which sharper curves, and angles close to $90^{\circ}$, are the "supernormal" patterns. This is entirely consistent with the LTG/NP approach, and shows that the visual system tends to rectify or "normalize" color-contour contingencies, essentially in terms of the primitive Lie orbits.

The present results are consistent with Stromeyer's (1978) statement that CAEs may be subserved by only simple patterns. However, the specific patterns defined as "simple" are a function of one's theoretical viewpoint, and the present experiments demonstrate that, at least to a first approximation, the LTG/NP has predictive power not evidenced by other theories in investigating the possible mechanisms of contingent aftereffects in human vision.

\section{REFERENCES}

Breitmeyer, B. G., \& COOPER, L. A. (1972). Frequency-specific color adaptation in the human visual system. Perception \& Psychophysics, 11, 95-96.

Caelli, T., Donwell, P. (1982). The discrimination of structure in vectorgraphs: Local and global effects. Perception \& Psychophysics, 32, 314-326.

Crassini, B., Broerse, J., \& O'SheA, R. P. (1979). Is the "contour specificity" of McCollough effects an example of anomalous transfer? Comments on Sharpe and Tees (1978). Perception \& Psychophysics, 25, 238-240.

Crassini, B., \& OVER, R. (1975). Curvature-specific color aftereffects. Perception \& Psychophysics, 17, 398-404.

DoDWELL, P. C. (1970a). Anomalous transfer effects after pattern discrimination training in rats and squirrels. Journal of Comparative and Physiological Psychology, 71, 42-51.

DoDWELl, P. C. (1970b). Visual pattern recognition. Toronto: Holt, Rinehart \& Winston.

DoDWELl, P. C. (1978). Human pattern and object perception. In R. Held, H. W. Leibowitz, \& H. L. Teuber (Eds.), Perception: Handbook of sensory physiology (Vol. 8). New York: Springer.

DODWELL, P. C. (1983). The Lie transformation group model of visual perception. Perception \& Psychophysics, 34, 1-16.

Dodwell, P. C., \& CAELli, T. (1985). Recognition of vectorgraphs under transformations: Local and global determinants. Quarterly Journal of Experimental Psychology, 37A, 1-23.

FIDELL, L. K. S. (1968). Pattem-specific complementary hue aftereffects: More on the McCollough-phenomenon. Unpublished doctoral dissertation, University of Michigan.

FIDELL, L. S. (1970). Orientation specificity in chromatic adaptation of human "edge-detectors." Perception \& Psychophysics, 8, 235-247.

Green, M., Corwin, T., Zemon, V. (1976). A comparison of Fourier analysis and feature analysis in pattern-specific color aftereffects. Science, 192, 147-148.

HaRRIs, C. S., \& Gibson, A. R. (1968). Is orientation-specific color 
adaptation in human vision due to edge detectors, afterimages, or " dipoles''? Science, 162, 1506-1507.

Hofrman, W. C. (1966). The Lie algebra of visual perception. Journal of Mathematical Psychology, 3, 65-98.

HoFrman, W. C. (1977). An informal, historical description (with bibliography) of the "L.T.G./N. P." Cahiers de Psychologies, 20, 135-174.

HubeL, D. H., \& WIESEL, T. N. (1962). Receptive fields, binocular interaction and functional architecture in the cat's visual cortex. Joumal of Physiology (London), 160, 106-154.

Hubel, D. H., \& WiESEL, T. N. (1968). Receptive fields and functional architecture of monkey striate cortex. Journal of Physiology (London), 195, 215-243.

JoNEs, P. D., \& HoLDING, D. H. (1975). Extremely long-term persistence of the McCollough effect. Journal of Experimental Psychology: Human Perception and Performance, 1, 323-327.

JoRDAN, K., \& UHLARIK, J. (1983). Contextual influences on a spatialfrequency-contingent color aftereffect. Perception \& Psychophysics, $33,420-424$

LEPPMANN, P. K. (1973). Spatial frequency dependent chromatic aftereffects. Nature, 242, 411-412.

LIE, S., \& ENGELS, F. (1888). Theorie der Transformationsgruppen. Leipzig: Teuber.

LOVEGROVE, W. J., \& OVER, R. (1972). Color adaptation of spatial frequency detectors in the human visual system. Science, 176, 541-543.

MACH, E. (1959). The analysis of sensations. New York: Dover. (Original work published 1886)

MACKAY, V., MACKAY, D. M. (1977). Multiple orientation-contingent chromatic aftereffects. Quarterly Journal of Experimental Psychology, 29, 203-218.

MARR, D. (1982). Vision. San Francisco: Freeman.

MAY, J. G., \& MATTESON, H. H. (1976). Spatial frequency-contingent color aftereffects. Science, 192, 145-147.

MCCollough, C. (1965). Color adaptation of edge-detectors in the human visual system. Science, 149, 1115-1116.

O'SheA, R. P., \& DoDwell, P. C. (1984, April-May). Local and 'global' influences on the McCollough effect. Paper presented at the meeting of the Association for Research in Vision and Ophthalmology, Sarasota, Florida.

Riggs, L. A. (1973). Curvature as a feature of pattern vision. Science, 181, 1070-1072.

Sharpe, L. T., \&ees, R. C. (1978). Contour specificity of the McCollough effect. Perception \& Psychophysics, 23, 451-458.
Sigel, C., \& NaCHMiAs, J. (1975). A re-evaluation of curvature-specific chromatic aftereffects. Vision Research, 15, 829-836.

Skowbo, D., Gentry, T., Timney, B., \& Morant, R. B. (1974). The McCollough effect: Influence of several kinds of visual stimulation on decay rate. Perception \& Psychophysics, 16, 47-49.

SмITH, F. D. (1977). Checkerboards and color aftereffects. [Letter to the editor]. Science, 198, 207-208.

STROMEYER, C. F., III. (1972a). Contour-contingent color aftereffects: Retinal area specificity. American Journal of Psychology, 85, 227-235.

STROMEYER, C. F., III. (1972b). Edge-contingent color aftereffects: spatial frequency specificity. Vision Research, 12, 717-733.

STROMEYER, C. F. (1978). Form-color aftereffects in human vision. In R. Held, H. W. Leibowitz, \& H. L. Teuber (Eds.), Perception: Handbook of sensory physiology (Vol. 8). New York: Springer.

TefT, L. W., \& ClaRK, F. T. (1968). The effects of stimulus density on orientation specific aftereffects of color adaptation. Psychonomic Science, 11, 265-266

TYLER, C. W. (1977). Checkerboards and color aftereffects [Letter to the editor]. Science, 198, 208-209.

WhITE, K. D., \& RigGs, L. A. (1974). Angle-contingent color aftereffects. Vision Research, 14, 1147-1154.

WyaTT, H. J. (1974). Singly and doubly contingent after-effects involving color, orientation, and spatial frequency. Vision Research, 14, 1185-1193.

\section{NOTES}

1. The use of steady fixation is important to the development of CAEs under the conditions reported here. In a pilot study, subjects whose gaze was allowed to wander over the patterns did not report CAEs except with the grating pair. However, O'Shea and Dodwell (1984) have recently demonstrated CAEs when eye movements are regulated. The point could be raised that the CAEs reported here are simple colored afterimages which are relatively specific, but the report of Harris and Gibson (1968), in combination with the results of the present Experiment 2 , argue strongly against this interpretation. Rather, it may be that central fixation is a necessary condition for adaption to vectorfields which are radially symmetric.

2. Copies of these raw data are available to readers for the cost of photocopying and postage (currently about $\$ 45.00$ Canadian).

(Manuscript received August 16, 1983; revision accepted for publication February 4, 1985.) 